\title{
A CHARACTERIZATION OF BARYCENTRICALLY PREASSOCIATIVE FUNCTIONS
}

\author{
JEAN-LUC MARICHAL AND BRUNO TEHEUX
}

\begin{abstract}
We provide a characterization of the variadic functions which are barycentrically preassociative as compositions of length-preserving associative string functions with one-to-one unary maps. We also discuss some consequences of this characterization.
\end{abstract}

\section{INTRODUCTION}

Let $X$ and $Y$ be arbitrary nonempty sets. Throughout this paper we regard tuples $\mathbf{x}$ in $X^{n}$ as $n$-strings over $X$. We let $X^{*}=\cup_{n \geqslant 0} X^{n}$ be the set of all strings over $X$, with the convention that $X^{0}=\{\varepsilon\}$ (i.e., $\varepsilon$ denotes the unique 0 -string on $X$ ). We denote the elements of $X^{*}$ by bold roman letters $\mathbf{x}, \mathbf{y}, \mathbf{z}$. If we want to stress that such an element is a letter of $X$, we use non-bold italic letters $x, y, z$, etc. The length of a string $\mathbf{x}$ is denoted by $|\mathbf{x}|$. For instance, $|\varepsilon|=0$. We endow the set $X^{*}$ with the concatenation operation, for which $\varepsilon$ is the neutral element, i.e., $\varepsilon \mathbf{x}=\mathbf{x} \varepsilon=\mathbf{x}$. For instance, if $\mathbf{x} \in X^{m}$ and $y \in X$, then $\mathbf{x} y \in X^{m+1}$. Moreover, for every string $\mathbf{x}$ and every integer $n \geqslant 0$, the power $\mathbf{x}^{n}$ stands for the string obtained by concatenating $n$ copies of $\mathbf{x}$. In particular we have $\mathbf{x}^{0}=\varepsilon$.

As usual, a map $F: X^{n} \rightarrow Y$ is said to be an $n$-ary function (an n-ary operation on $X$ if $Y=X$ ). Also, a map $F: X^{*} \rightarrow Y$ is said to be a variadic function (a string function on $X$ if $Y=X^{*}$; see [5]). For every variadic function $F: X^{*} \rightarrow Y$ and every integer $n \geqslant 0$, we denote by $F_{n}$ the $n$-ary part $\left.F\right|_{X^{n}}$ of $F$.

Recall that a variadic function $F: X^{*} \rightarrow Y$ is said to be preassociative [6,7] if, for any $\mathbf{x}, \mathbf{y}, \mathbf{y}^{\prime}, \mathbf{z} \in X^{*}$, we have

$$
F(\mathbf{y})=F\left(\mathbf{y}^{\prime}\right) \quad \Rightarrow \quad F(\mathbf{x y z})=F\left(\mathbf{x y}^{\prime} \mathbf{z}\right) .
$$

Also, a variadic function $F: X^{*} \rightarrow Y$ is said to be barycentrically preassociative (or $B$ preassociative for short) [8] if, for any $\mathbf{x}, \mathbf{y}, \mathbf{y}^{\prime}, \mathbf{z} \in X^{*}$, we have

$$
|\mathbf{y}|=\left|\mathbf{y}^{\prime}\right| \quad \text { and } F(\mathbf{y})=F\left(\mathbf{y}^{\prime}\right) \quad \Rightarrow \quad F(\mathbf{x y z})=F\left(\mathbf{x y}^{\prime} \mathbf{z}\right) .
$$

Contrary to preassociativity, B-preassociativity recalls the associativity-like property of the barycenter (just regard $F(\mathbf{x})$ as the barycenter of a set $\mathbf{x}$ of identical homogeneous balls in $X=\mathbb{R}^{n}$ ). In descriptive statistics and aggregation function theory, this condition says that the aggregated value of a series of numerical values remains unchanged when modifying a bundle of these values without changing their partial aggregation.

B-preassociativity has been recently utilized by the authors in the following characterization of the quasi-arithmetic pre-mean functions, thus generalizing the well-known Kolmogoroff-Nagumo's characterization of the quasi-arithmetic mean functions.

Date: September 22, 2015.

2010 Mathematics Subject Classification. 39B72.

Key words and phrases. Associativity, barycentric associativity, barycentric preassociativity, string functions, functional equation, axiomatization. 
Theorem 1 ( [8]). Let $\mathbb{I}$ be a nontrivial real interval, possibly unbounded. A function $F: \mathbb{I}^{*} \rightarrow \mathbb{R}$ is B-preassociative and, for every $n \geqslant 1$, the function $F_{n}$ is symmetric, continuous, and strictly increasing in each argument if and only if there are continuous and strictly increasing functions $f: \mathbb{I} \rightarrow \mathbb{R}$ and $f_{n}: \mathbb{R} \rightarrow \mathbb{R}(n \geqslant 1)$ such that

$$
F_{n}(\mathbf{x})=f_{n}\left(\frac{1}{n} \sum_{i=1}^{n} f\left(x_{i}\right)\right), \quad n \geqslant 1 .
$$

Remark 1 . If we add the condition that every $F_{n}$ is idempotent (i.e., $F_{n}\left(x^{n}\right)=x$ for every $x \in X$ ) in Theorem 1, then we necessarily have $f_{n}=f^{-1}$ for every $n \geqslant 1$, thus reducing this result to Kolmogoroff-Nagumo's characterization of the quasi-arithmetic mean functions [4] 9]. However, there are also many non-idempotent quasi-arithmetic pre-mean functions. Taking for instance $f_{n}(x)=n x$ and $f(x)=x$ over the reals $\mathbb{I}=\mathbb{R}$, we obtain the sum function. Taking $f_{n}(x)=\exp (n x)$ and $f(x)=\ln (x)$ over $\left.\mathbb{I}=\right] 0, \infty[$, we obtain the product function.

In this paper we show that B-preassociative functions can be factorized as compositions of length-preserving associative string functions with one-to-one unary maps. We also show how this factorization result generalizes a characterization of a noteworthy subclass of B-preassociative functions given by the authors in [8]. Finally, we mention some interesting consequences of this new characterization.

The terminology used throughout this paper is the following. The domain, range, and kernel of any function $f$ are denoted by $\operatorname{dom}(f), \operatorname{ran}(f)$, and $\operatorname{ker}(f)$, respectively. The identity function on any nonempty set is denoted by id. For every $n \geqslant 1$, the diagonal section $\delta_{F}: X \rightarrow Y$ of a function $F: X^{n} \rightarrow Y$ is defined as $\delta_{F}(x)=F\left(x^{n}\right)$.

Remark 2. Although B-preassociativity was recently defined by the authors [8], the basic idea behind this definition goes back to 1931 when de Finetti [1] introduced an associativitylike property for mean functions. Indeed, according to de Finetti, for a real function $F: \cup_{n \geqslant 1} \mathbb{R}^{n} \rightarrow \mathbb{R}$ to be considered as a mean, it is natural that it be "associative" in the following sense: for any $u \in X$ and any $\mathbf{x}, \mathbf{y}, \mathbf{z} \in X^{*}$ such that $|\mathbf{x z}| \geqslant 1$ and $|\mathbf{y}| \geqslant 1$, we have $F(\mathbf{x y z})=F\left(\mathbf{x} u^{|\mathbf{y}|} \mathbf{z}\right)$ whenever $F(\mathbf{y})=F\left(u^{|\mathbf{y}|}\right)$.

\section{MAIN RESULTS}

As mentioned in the introduction, in this section we mainly show that B-preassociative functions can be factorized as compositions of length-preserving associative string functions with one-to-one unary maps. This result is stated in Theorem 8

Recall that a string function $F: X^{*} \rightarrow X^{*}$ is said to be associative [5] if it satisfies the equation $F(\mathbf{x y z})=F(\mathbf{x} F(\mathbf{y}) \mathbf{z})$ for any $\mathbf{x}, \mathbf{y}, \mathbf{z} \in X^{*}$.

Definition 2. We say that a string function $F: X^{*} \rightarrow X^{*}$ is length-preserving if $|F(\mathrm{x})|=$ $|\mathbf{x}|$ for every $\mathbf{x} \in X^{*}$, or equivalently, if $\operatorname{ran}\left(F_{n}\right) \subseteq X^{n}$ for every $n \geqslant 0$.

Clearly, the identity function on $X^{*}$ is associative and length-preserving. The following example gives nontrivial instances of associative and length-preserving string functions. Further examples of associative string functions can be found in [5].

Example 3. Let $\left(h_{n}\right)_{n \geqslant 1}$ be a sequence of unary operations on $X$. One can easily see that the length-preserving function $F: X^{*} \rightarrow X^{*}$ defined by $F_{0}(\varepsilon)=\varepsilon$ and

$$
F_{n}\left(x_{1} \cdots x_{n}\right)=h_{1}\left(x_{1}\right) \cdots h_{n}\left(x_{n}\right), \quad n \geqslant 1,
$$

is associative if and only if $h_{n} \circ h_{m}=h_{n}$ for all $n, m \geqslant 1$ such that $m \leqslant n$. Using an elementary induction, one can also show that the latter condition is equivalent to $h_{n} \circ h_{n}=$ 
$h_{n}$ and $h_{n+1} \circ h_{n}=h_{n+1}$ for every $n \geqslant 1$. To give an example, take any constant sequence $h_{n}=h$ such that $h \circ h=h$ (for instance, the positive part function $h(x)=x^{+}$over $X=\mathbb{R}$ ). As a second example, consider the sequence $h_{n}$ of unary operations on $X=\{1,2,3, \ldots\}$ defined by $h_{n}(k)=1$ if $k \leqslant n+1$, and $h_{n}(k)=k$, otherwise.

Proposition 4. Let $F: X^{*} \rightarrow X^{*}$ be a length-preserving function. Then $F$ is associative if and only if it is B-preassociative and satisfies $F_{n}=F_{n} \circ F_{n}$ for every $n \geqslant 0$.

Proof. To see that the necessity holds, we recall from [5] that any associative string function is preassociative and hence B-preassociative. The second part of the statement is immediate. For the sufficiency, we merely observe that we have $F(F(\mathbf{y}))=F(\mathbf{y})$ for every $\mathbf{y} \in X^{*}$ and therefore, by B-preassociativity, we also have $F(\mathbf{x} F(\mathbf{y}) \mathbf{z})=F(\mathbf{x y z})$ for every $\mathbf{x y z} \in X^{*}$, that is, $F$ is associative.

The following proposition, established in [8], shows how we can construct new Bpreassociative functions from given B-preassociative functions.

Proposition 5 ( [8]). Let $F: X^{*} \rightarrow Y$ be a B-preassociative function and let $\left(g_{n}\right)_{n \geqslant 1}$ be a sequence of functions from $Y$ to a nonempty set $Y^{\prime}$. If $\left.g_{n}\right|_{\operatorname{ran}\left(F_{n}\right)}$ is one-to-one for every $n \geqslant 1$, then any function $H: X^{*} \rightarrow Y^{\prime}$ such that $H_{n}=g_{n} \circ F_{n}$ for every $n \geqslant 1$ is $B$-preassociative.

Recall that a function $g$ is a quasi-inverse [10, Sect. 2.1] of a function $f$ if

$$
\left.f \circ g\right|_{\operatorname{ran}(f)}=\left.\mathrm{id}\right|_{\operatorname{ran}(f)} \quad \text { and } \quad \operatorname{ran}\left(\left.g\right|_{\operatorname{ran}(f)}\right)=\operatorname{ran}(g) \text {. }
$$

We denote the set of quasi-inverses of a function $f$ by $Q(f)$. Under the assumption of the Axiom of Choice (AC), the set $Q(f)$ is nonempty for any function $f$. In fact, the Axiom of Choice is just another form of the statement "every function has a quasi-inverse". Note also that the relation of being quasi-inverse is symmetric: if $g \in Q(f)$ then $f \in Q(g)$; moreover, we have $\operatorname{ran}(g) \subseteq \operatorname{dom}(f)$ and $\operatorname{ran}(f) \subseteq \operatorname{dom}(g)$ and the functions $\left.f\right|_{\operatorname{ran}(g)}$ and $\left.g\right|_{\operatorname{ran}(f)}$ are one-to-one.

Lemma 6. Assume AC and let $F: X^{n} \rightarrow Y$ be a function. For any $g \in Q(F)$, define the function $H: X^{n} \rightarrow X^{n}$ by $H=g \circ F$. Then we have $F=F \circ H$ and $H=H \circ H$. Moreover, the map $\left.F\right|_{\operatorname{ran}(H)}$ is one-to-one.

Proof. By definition of $H$ we have $F \circ H=F \circ g \circ F=F$ and $H \circ H=g \circ F \circ g \circ F=$ $g \circ F=H$. Also, the map $\left.F\right|_{\operatorname{ran}(H)}=\left.F\right|_{\operatorname{ran}(g)}$ is one-to-one.

Lemma 7. Assume $A C$ and let $F: X^{*} \rightarrow Y$ be a function. The following assertions are equivalent.

(i) $F$ is B-preassociative.

(ii) For every sequence $\left(g_{n} \in Q\left(F_{n}\right)\right)_{n \geqslant 1}$, the function $H: X^{*} \rightarrow X^{*}$ defined by $H_{0}(\varepsilon)=\varepsilon$ and $H_{n}=g_{n} \circ F_{n}$ for every $n \geqslant 1$ is associative and length-preserving.

(iii) There exists a sequence $\left(g_{n} \in Q\left(F_{n}\right)\right)_{n \geqslant 1}$ such that the function $H: X^{*} \rightarrow X^{*}$ defined by $H_{0}(\varepsilon)=\varepsilon$ and $H_{n}=g_{n} \circ F_{n}$ for every $n \geqslant 1$ is associative and lengthpreserving.

Proof. (i) $\Rightarrow$ (ii). Let $H: X^{*} \rightarrow X^{*}$ be defined as indicated in the statement. We know by Lemma 6 that $H \circ H=H$ and $H$ is length-preserving. Since $\left.g_{n}\right|_{\operatorname{ran}\left(F_{n}\right)}$ is one-to-one, we have that $H$ is B-preassociative by Proposition 5 It follows from Proposition 4 that $H$ is associative.

(ii) $\Rightarrow$ (iii). Trivial. 
(iii) $\Rightarrow$ (i). By Proposition $4, H$ is B-preassociative. For every $n \geqslant 1$, since $\left.g_{n}\right|_{\operatorname{ran}\left(F_{n}\right)}$ is a one-to-one map from $\operatorname{ran}\left(F_{n}\right)$ onto $\operatorname{ran}\left(g_{n}\right)=\operatorname{ran}\left(H_{n}\right)$, we have $F_{n}=\left(\left.g_{n}\right|_{\operatorname{ran}\left(F_{n}\right)}\right)^{-1}$ 。 $H_{n}$. By Proposition 5 it follows that $F$ is B-preassociative.

We are now ready to present our main result, which gives a characterization of any Bpreassociative function as a composition of a length-preserving associative string function with one-to-one unary maps.

Theorem 8. Assume AC and let $F: X^{*} \rightarrow Y$ be a function. The following assertions are equivalent.

(i) $F$ is B-preassociative.

(ii) There exist an associative and length-preserving function $H: X^{*} \rightarrow X^{*}$ and a sequence $\left(f_{n}\right)_{n \geqslant 1}$ of one-to-one functions $f_{n}: \operatorname{ran}\left(H_{n}\right) \rightarrow Y$ such that $F_{n}=f_{n}$ 。 $H_{n}$ for every $n \geqslant 1$.

If condition (ii) holds, then for every $n \geqslant 1$ we have $f_{n}=\left.F\right|_{\operatorname{ran}\left(H_{n}\right)}=\left.F_{n}\right|_{\operatorname{ran}\left(H_{n}\right)}, f_{n}^{-1} \epsilon$ $Q\left(F_{n}\right)$, and we may choose $H_{n}=g_{n} \circ F_{n}$ for any $g_{n} \in Q\left(F_{n}\right)$.

Proof. (i) $\Rightarrow$ (ii). Let $H: X^{*} \rightarrow X^{*}$ be defined by $H_{0}(\varepsilon)=\varepsilon$ and $H_{n}=g_{n} \circ F_{n}$ for every $n \geqslant 1$, where $g_{n} \in Q\left(F_{n}\right)$. By Lemma 6 we have $F_{n}=f_{n} \circ H_{n}$ for every $n \geqslant 1$, where $f_{n}=\left.F_{n}\right|_{\operatorname{ran}\left(H_{n}\right)}$ is one-to-one. By Lemma7 $H$ is associative and length-preserving.

(ii) $\Rightarrow$ (i). $H$ is B-preassociative by Proposition 4 . By Proposition 5 it follows that also $F$ is B-preassociative.

If condition (ii) holds, then for every $n \geqslant 1$ we have $F_{n} \circ H_{n}=f_{n} \circ H_{n} \circ H_{n}=f_{n} \circ H_{n}$ and hence $\left.F_{n}\right|_{\operatorname{ran}\left(H_{n}\right)}=f_{n}$. Moreover, since $f_{n}$ is one-to-one, we have $H_{n}=f_{n}^{-1} \circ F_{n}$ and hence $F_{n} \circ f_{n}^{-1} \circ F_{n}=F_{n} \circ H_{n}=f_{n} \circ H_{n} \circ H_{n}=f_{n} \circ H_{n}=F_{n}$, which shows that $f_{n}^{-1} \in Q\left(F_{n}\right)$.

Remark 3. (a) It is clear that the trivial factorization $F_{n}=F_{n} \circ H_{n}$, where $H_{n}=$ id, holds for any function $F: X^{*} \rightarrow Y$. This observation could make us wrongly think that Theorem 8 is of no use. However, in our factorization $F_{n}=f_{n} \circ H_{n}$ the outer function $f_{n}$ has the important feature that it is one-to-one.

(b) Similarly to Theorem 8 , one can show [5] that any preassociative function $F: X^{*} \rightarrow$ $Y$ can be factorized as a composition $F=f \circ H$, where $H: X^{*} \rightarrow X^{*}$ is associative and $f: \operatorname{ran}(H) \rightarrow Y$ is one-to-one.

In the rest of this section we show how Theorem 8 can be particularized to some nested subclasses of B-preassociative functions, including the subclass of B-preassociative functions $F: X^{*} \rightarrow Y$ for which the equality $\operatorname{ran}\left(F_{n}\right)=\operatorname{ran}\left(\delta_{F_{n}}\right)$ holds for every $n \geqslant 1$ (see [8]).

For any integers $m, n \geqslant 1$, define $X_{m}^{0}=X^{0}$ and

$$
X_{m}^{n}=\left\{\mathbf{y} z^{n-\min \{n, m\}+1}: \mathbf{y} z \in X^{\min \{n, m\}}\right\} .
$$

For instance $X_{1}^{3}=\left\{z^{3}: z \in X\right\}, X_{2}^{3}=\left\{y z^{2}: y z \in X^{2}\right\}$, and $X_{m}^{3}=X^{3}$ for every $m \geqslant 3$.

Thus, we have $X_{m}^{n}=X^{n}$ if $m \geqslant n$ and $X_{m}^{n}=\left\{\mathbf{y} z^{n-m+1}: \mathbf{y} z \in X^{m}\right\}$ if $m \leqslant n$. It follows that for every $m \geqslant 1$ we have $X_{m}^{n} \subseteq X_{m+1}^{n} \subseteq X^{n}$.

Definition 9. Let $m \geqslant 1$ and $n \geqslant 0$ be integers. We say that a function $H: X^{n} \rightarrow X^{n}$ has an $m$-generated range if $\operatorname{ran}(H) \subseteq X_{m}^{n}$. We say that a function $H: X^{*} \rightarrow X^{*}$ has an $m$-generated range if $H_{n}$ has an $m$-generated range for every $n \geqslant 0$. 
Fact 10. If a function $H: X^{n} \rightarrow X^{n}$ has an m-generated range, then it has an $(m+1)$ generated range. If a function $H: X^{*} \rightarrow X^{*}$ has an m-generated range, then it is lengthpreserving.

Let $m \geqslant 1$ and $n \geqslant 0$ be integers. The $m$-diagonal section of a function $F: X^{n} \rightarrow Y$ is the map $\delta_{F}^{m}: X^{\min \{n, m\}} \rightarrow Y$ defined by $\delta_{F}^{m}=F$, if $n=0$, and $\delta_{F}^{m}(\mathbf{y} z)=F\left(\mathbf{y} z^{n-\min \{n, m\}+1}\right)$ for every $\mathbf{y} z \in X^{\min \{n, m\}}$, otherwise. We clearly have $\operatorname{ran}\left(\delta_{F}^{m}\right) \subseteq \operatorname{ran}\left(\delta_{F}^{m+1}\right)$.

Definition 11. Let $m \geqslant 1$ and $n \geqslant 0$ be integers. We say that a function $F: X^{n} \rightarrow Y$ is m-quasi-range-idempotent if $\operatorname{ran}(F)=\operatorname{ran}\left(\delta_{F}^{m}\right)$.

By definition, any $m$-quasi-range-idempotent function $F: X^{n} \rightarrow Y$ is $(m+1)$-quasirange-idempotent. We also observe that the property of being $m$-quasi-range-idempotent is preserved under left composition with unary maps: if $F: X^{n} \rightarrow Y$ is $m$-quasi-rangeidempotent, then so is $g \circ F$ for any map $g: Y \rightarrow Y^{\prime}$, where $Y^{\prime}$ is a nonempty set.

Proposition 12. If $F: X^{*} \rightarrow X^{*}$ is associative and $F_{k}$ has an $m$-generated range for some $k, m \geqslant 1$, then for any integer $p \geqslant 0$ the function $F_{k+p}$ is $(m+p)$-quasi-range-idempotent. In particular, $F_{k}$ is m-quasi-range-idempotent.

Proof. Let $\mathbf{x} \in X^{p}$ and $\mathbf{x}^{\prime} \in X^{k}$. Then, there exists $\mathbf{y} z \in X^{\min \{k, m\}}$ such that

$$
\begin{aligned}
F_{k+p}\left(\mathbf{x x}^{\prime}\right) & =F_{k+p}\left(\mathbf{x} F_{k}\left(\mathbf{x}^{\prime}\right)\right)=F_{k+p}\left(\mathbf{x y} z^{k-\min \{k, m\}+1}\right) \\
& =F_{k+p}\left(\mathbf{x y} z^{(k+p)-\min \{k+p, m+p\}+1}\right)=\delta_{F_{k+p}}^{m+p}(\mathbf{x y} z),
\end{aligned}
$$

which shows that $\operatorname{ran}\left(F_{k+p}\right) \subseteq \operatorname{ran}\left(\delta_{F_{k+p}}^{m+p}\right)$. The converse inclusion is obvious.

Lemma 13. Let $m, n \geqslant 1$ be integers. Any map $F: X^{n} \rightarrow Y$ satisfying $F=F \circ H$, where $H: X^{n} \rightarrow X^{n}$ has an m-generated range, is m-quasi-range-idempotent.

Proof. Since $\operatorname{ran}(H) \subseteq X_{m}^{n}$, we have $\operatorname{ran}(F)=\operatorname{ran}(F \circ H) \subseteq \operatorname{ran}\left(\delta_{F}^{m}\right)$. Since the converse inclusion $\operatorname{ran}(F) \supseteq \operatorname{ran}\left(\delta_{F}^{m}\right)$ holds for any map $F: X^{n} \rightarrow Y$, we have that $F$ is $m$-quasi-range-idempotent.

Lemma 14. Under the assumptions of Lemma 6 if $F$ is m-quasi-range-idempotent for some $m \geqslant 1$, then $g$ can always be chosen so that $\operatorname{ran}(g) \subseteq X_{m}^{n}$ and therefore $H$ has an $m$-generated range. Conversely, if $H$ has an $m$-generated range for some $m \geqslant 1$, then $F$ is m-quasi-range-idempotent.

Proof. If $F$ is $m$-quasi-range-idempotent for some $m \geqslant 1$, then there always exists $g \in$ $Q(F)$ such that $\operatorname{ran}(g) \subseteq X_{m}^{n}$; indeed, if $y \in \operatorname{ran}(F)=\operatorname{ran}\left(\delta_{F}^{m}\right)$, then we can take $g(y) \in\left(\delta_{F}^{m}\right)^{-1}\{y\} \subseteq X_{m}^{n}$. Therefore $H=g \circ F$ has an $m$-generated range. Conversely, if $H$ has an $m$-generated range for some $m \geqslant 1$, then $F$ is $m$-quasi-range-idempotent by Lemma 13 .

Corollary 15. For any $m \geqslant 1$, the equivalence in Lemma 7 holds if we add the condition that every $F_{n}(n \geqslant 1)$ is m-quasi-range-idempotent in assertion ( $\left.i\right)$ and the conditions that $\operatorname{ran}\left(g_{n}\right) \subseteq X_{m}^{n}(n \geqslant 1)$ and $H$ has an m-generated range in assertions (ii) and (iii).

Theorem 16. For any $m \geqslant 1$, the equivalence between (i) and (ii) in Theorem 8 still holds if we add the condition that every $F_{n}(n \geqslant 1)$ is m-quasi-range-idempotent in assertion (i) and the condition that $H$ has an m-generated range in assertion (ii). In this case the condition $\operatorname{ran}\left(g_{n}\right) \subseteq X_{m}^{n}(n \geqslant 1)$ must be added in the last part of the statement.

Proof. Follows from the results above. 
Setting $m=1$ in Theorem 16 , we immediately derive a factorization of any B-preassociative function whose $n$-ary part $F_{n}$ is 1-quasi-range-idempotent for every $n \geqslant 1$. An alternative factorization for such functions is given in the following theorem, established in [8]. Recall that a function $F: X^{*} \rightarrow X \cup\{\varepsilon\}$ is barycentrically associative (or $B$ associative for short) [8] if it satisfies the equation $F(\mathbf{x y z})=F\left(\mathbf{x} F(\mathbf{y})^{|\mathbf{y}|} \mathbf{z}\right)$ for any $\mathbf{x}, \mathbf{y}, \mathbf{z} \in X^{*}$. (B-associativity is also known as decomposability, see [2,3]).

Theorem 17 ( [8]). Assume AC and let $F: X^{*} \rightarrow Y$ be a function. The following assertions are equivalent.

(i) $F$ is B-preassociative and $F_{n}$ is 1-quasi-range-idempotent for every $n \geqslant 1$.

(ii) There exists a B-associative function $H: X^{*} \rightarrow X \cup\{\varepsilon\}$ such that $H(\varepsilon)=\varepsilon$ and a sequence $\left(f_{n}\right)_{n \geqslant 1}$ of one-to-one functions $f_{n}: \operatorname{ran}\left(H_{n}\right) \rightarrow Y$ such that $F_{n}=$ $f_{n} \circ H_{n}$ for every $n \geqslant 1$.

If condition (ii) holds, then for every $n \geqslant 1$ we have $F_{n}=\delta_{F_{n}} \circ H_{n}, f_{n}=\delta_{F_{n}} \mid \operatorname{ran}\left(H_{n}\right)$, $f_{n}^{-1} \in Q\left(\delta_{F_{n}}\right)$, and we may choose $H_{n}=g_{n} \circ F_{n}$ for any $g_{n} \in Q\left(\delta_{F_{n}}\right)$.

We now show how Theorem 17 can be easily derived from Theorem 16 .

For every $m \geqslant 1$ and every $\mathbf{x} \in X^{*}$, denote by $\mathbf{x}_{[m]}$ the $m$-prefix of $\mathbf{x}$, that is the string in $\bigcup_{i=0}^{m} X^{i}$ defined as follows: if $|\mathbf{x}| \leqslant m$, then $\mathbf{x}_{[m]}=\mathbf{x}$; otherwise, if $\mathbf{x}=\mathbf{x}^{\prime} \mathbf{x}^{\prime \prime}$, with $\left|\mathbf{x}^{\prime}\right|=m$, then $\mathbf{x}_{[m]}=\mathbf{x}^{\prime}$.

If $H: X^{*} \rightarrow X^{*}$ has an $m$-generated range, then by definition it can be assimilated with the function $H_{[m]}: X^{*} \rightarrow \bigcup_{i=0}^{m} X^{i}$ defined by $H_{[m]}(\mathbf{x})=H(\mathbf{x})_{[m]}$. Indeed, $H$ can be reconstructed from $H_{[m]}$ by setting

$$
H(\mathbf{x})= \begin{cases}H_{[m]}(\mathbf{x}), & \text { if }|\mathbf{x}| \leqslant m, \\ H_{[m]}(\mathbf{x}) z^{n-m}, & \text { otherwise }\end{cases}
$$

where $z$ is the last letter of $H_{[m]}(\mathbf{x})$.

Thus we can prove Theorem 17 from Theorem 16 as follows.

Proof of Theorem 17 as a corollary of Theorem 16 By setting $m=1$ in Theorem 16, we see that $H$ has a 1-generated range. By the observation above, $H$ can then be assimilated with $H_{[1]}$ through the identity $H(\mathbf{x})=H_{[1]}(\mathbf{x})^{|\mathbf{x}|}$ for every $\mathbf{x} \in X^{*}$. It is then clear that $H$ is associative if and only if $H_{[1]}$ is $\mathrm{B}$-associative. The other parts of Theorem 17 follow immediately.

Remark 4. The question of generalizing Theorem 17 by dropping the 1-quasi-range-idempotent condition on every $F_{n}$ was raised in [8]. Clearly, Theorem 8 answers this question.

\section{SOME CONSEQUENCES OF THE FACTORIZATION RESULT}

Since any associative function $F: X^{*} \rightarrow X^{*}$ is preassociative and, in turn, B-preassociative, it can be factorized as indicated in Theorem 8 . Therefore, up to one-to-one unary maps, the associative string functions can be completely described in terms of lengthpreserving associative string functions, and similarly for the preassociative and B-preassociative functions. This is an important observation which shows that in a sense any of these nested classes can be described in terms of the smallest one, namely the subclass of associative and length-preserving string functions (see Figure 1).

Example 18. Let $a \in X$ be fixed. Let the map $F: X^{*} \rightarrow X^{*}$ be defined inductively by $F(z)=z$ if $z \neq a, F(a)=\varepsilon$, and $F(\mathbf{x} z)=F(\mathbf{x}) F(z)$ for every $\mathbf{x} z \in X^{*}$. Thus defined, $F(\mathbf{x})$ is obtained from $\mathbf{x}$ by removing all the 'a' letters (if any). Since $F$ is 


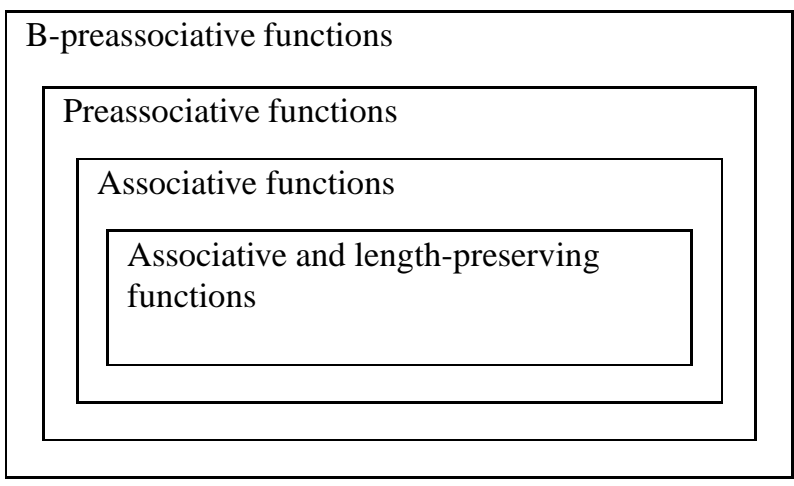

FIGURE 1. Nested subclasses of B-preassociative functions

associative (see [5] for more details), it is B-preassociative and therefore it can be factorized as indicated in Theorem 8 For every $n \geqslant 1$, define the function $g_{n}: \cup_{i=0}^{n}(X \backslash\{a\})^{i} \rightarrow X^{n}$ by $g_{n}(\mathbf{x})=\mathbf{x} a^{n-\mid \mathbf{x}}$. Since $F_{n} \circ g_{n} \circ F_{n}=F_{n}$ for every $n \geqslant 1$, we see that $g_{n} \in Q\left(F_{n}\right)$. By Theorem 8, the function $H: X^{*} \rightarrow X^{*}$, defined by $H_{0}(\varepsilon)=\varepsilon$ and $H_{n}=g_{n} \circ F_{n}$ for every $n \geqslant 1$, is associative and length-preserving. Moreover, we have $F_{n}=f_{n} \circ H_{n}$ for every $n \geqslant 1$, where $f_{n}=\left.F_{n}\right|_{\operatorname{ran}\left(H_{n}\right)}$. Thus defined, $H_{n}(\mathbf{x})$ is obtained from $\mathbf{x}$ by moving all the 'a' letters (if any) to the rightmost positions. For instance, $H_{11}$ (mathematics $)=$ mthemticsaa.

As observed in the previous section, setting $m=1$ in Theorem 16, we can derive a factorization of any B-preassociative function whose $n$-ary part $F_{n}$ is 1 -quasi-rangeidempotent for every $n \geqslant 1$ (Theorem 17). In the following example, we derive a similar factorization explicitly directly from Theorem 8 (without using Theorem [16).

Example 19. If we assume that $F_{n}$ is 1-quasi-range-idempotent for every $n \geqslant 1$ in assertion (i) of Theorem 8 , then the factorization given in assertion (ii) can be obtained by defining $H_{n}=g_{n} \circ F_{n}$, where $g_{n}(x)=h_{n}(x)^{n}$ and $h_{n} \in Q\left(\delta_{F_{n}}\right)$. Indeed, since $F_{n}$ is 1-quasirange-idempotent, we have

$$
\left(F_{n} \circ g_{n} \circ F_{n}\right)(\mathbf{x})=\left(\delta_{F_{n}} \circ h_{n} \circ F_{n}\right)(\mathbf{x})=F_{n}(\mathbf{x}),
$$

which shows that $g_{n} \in Q\left(F_{n}\right)$.

It is clear that the B-associativity property, originally defined for functions $F: X^{*} \rightarrow$ $X \cup\{\varepsilon\}$ can be immediately extended to string functions $F: X^{*} \rightarrow X^{*}$.

Definition 20. We say that a string function $F: X^{*} \rightarrow X^{*}$ is barycentrically associative (or $B$-associative for short) if it satisfies the equation $F(\mathbf{x y z})=F\left(\mathbf{x} F(\mathbf{y})^{|\mathbf{y}|} \mathbf{z}\right)$ for any $\mathbf{x}, \mathbf{y}, \mathbf{z} \in X^{*}$.

It is easy to see that any B-associative string function $F: X^{*} \rightarrow X^{*}$ is B-preassociative and hence can be factorized as indicated in Theorem 8 . Moreover, any B-associative string function satisfying $\operatorname{ran}\left(F_{n}\right) \subseteq X$ for every $n \geqslant 1$ is also such that $F_{n}$ is 1-quasi-rangeidempotent for every $n \geqslant 1$ (see $[8]$ ) and therefore it can be factorized as described in Example 19. In this case we have $\delta_{F_{n}} \circ F_{n}=F_{n}$, which shows that $\left.\operatorname{id}\right|_{\operatorname{ran}\left(F_{n}\right)} \in Q\left(\delta_{F_{n}}\right)$ for every $n \geqslant 1$. Therefore, from Example 19 we immediately derive the following corollary. 
Corollary 21. Let $F: X^{*} \rightarrow X^{*}$ be a B-associative function satisfying $\operatorname{ran}\left(F_{n}\right) \subseteq X$ for every $n \geqslant 1$. Then, for every $n \geqslant 1$, we have $F_{n}=f_{n} \circ H_{n}$, where $H: X^{*} \rightarrow X^{*}$ is the length-preserving associative function defined by $H_{n}(\mathbf{x})=F_{n}(\mathbf{x})^{n}$ for every $n \geqslant 1$ and $f_{n}: \operatorname{ran}\left(H_{n}\right) \rightarrow X$ is the one-to-one function defined by $f_{n}\left(x^{n}\right)=x$ for every $n \geqslant 1$.

We end this section by an additional application of Theorem 8 .

Definition 22. We say that a function $F: X^{*} \rightarrow Y$ has a componentwise defined kernel if there exists a family $\left\{E_{n}: n \geqslant 1\right\}$ of equivalence relations on $X$ such that for any $n \geqslant 1$ and any $\mathbf{x}, \mathbf{y} \in X^{n}$, we have $F(\mathbf{x})=F(\mathbf{y})$ if and only if $\left(x_{i}, y_{i}\right) \in E_{i}$ for $i=1, \ldots, n$. In this case, we say that the family $\left\{E_{n}: n \geqslant 1\right\}$ defines the kernel of $F$ componentwise.

This concept can be interpreted, e.g., in decision making, as follows. A function $F: X^{*} \rightarrow Y$ has a componentwise defined kernel if the equivalence between two $n$-profiles $\mathbf{x}, \mathbf{y} \in X^{n}$ can be defined attributewise.

The following proposition and corollary give characterizations of those B-preassociative functions which have a componentwise defined kernel.

Proposition 23. Assume AC and let $F: X^{*} \rightarrow Y$ have a kernel defined componentwise by the family $\left\{E_{n}: n \geqslant 1\right\}$ of equivalence relations on $X$. Then $F$ is B-preassociative if and only if $E_{n} \subseteq E_{n+1}$ for every $n \geqslant 1$.

Proof. Let $F: X^{*} \rightarrow Y$ be defined as indicated in the statement. For the necessity, suppose that $F$ is B-preassociative and let $(x, y) \in E_{n}$ for some $n \geqslant 1$. Then we have $F\left(x^{n}\right)=$ $F\left(x^{n-1} y\right)$ and hence $F\left(x^{n+1}\right)=F\left(x^{n} y\right)$ by B-preassociativity. It follows that $(x, y) \in$ $E_{n+1}$. For the sufficiency, for any $n \geqslant 1$ and any $\mathbf{x}, \mathbf{y} \in X^{n}$ such that $F(\mathbf{x})=F(\mathbf{y})$, we have $F(\mathbf{x z})=F(\mathbf{y z})$ for every $\mathbf{z} \in X^{*}$ by definition of $F$. Since $E_{n} \subseteq E_{n+1}$ for every $n \geqslant$ 1 , we also have $F(\mathbf{z x})=F(\mathbf{z y})$ for every $\mathbf{z} \in X^{*}$. Therefore $F$ is B-preassociative.

Corollary 24. Assume AC and let $F: X^{*} \rightarrow Y$ be a function. The following assertions are equivalent.

(i) F is B-preassociative and has a componentwise defined kernel.

(ii) There exists a sequence $\left(h_{n}\right)_{n \geqslant 1}$ of unary operations on $X$ and a sequence $\left(f_{n}\right)_{n \geqslant 1}$ of one-to-one maps $f_{n}:\left\{h_{1}\left(x_{1}\right) \cdots h_{n}\left(x_{n}\right): x_{1} \cdots x_{n} \in X^{n}\right\} \rightarrow Y$ such that $h_{n}$ 。 $h_{n}=h_{n}, h_{n+1} \circ h_{n}=h_{n+1}$, and $F_{n}(\mathbf{x})=f_{n}\left(h_{1}\left(x_{1}\right) \cdots h_{n}\left(x_{n}\right)\right)$ for every $n \geqslant 1$ and every $\mathrm{x} \in X^{n}$.

Proof. (i) $\Rightarrow$ (ii). By Proposition 23 , the kernel of $F$ is defined by some family of equivalence relations $\left\{E_{n}: n \geqslant 1\right\}$ on $X$ satisfying $E_{n} \subseteq E_{n+1}$ for every $n \geqslant 1$. For every $c \in X / E_{n}$, let $s_{n}(c) \in c$ be a representative of $c$ and define the map $h_{n}: X \rightarrow X$ by $h_{n}(x)=$ $s_{n}\left(x / E_{n}\right)$. The map $g_{n}: \operatorname{ran}\left(F_{n}\right) \rightarrow X^{n}$ defined by $g_{n}(F(\mathbf{x}))=h_{1}\left(x_{1}\right) \cdots h_{n}\left(x_{n}\right)$ is a quasi-inverse of $F_{n}$. Indeed, since $\left(x_{i}, h_{i}\left(x_{i}\right)\right) \in E_{i}$ for every $\mathbf{x} \in X^{n}$ and every $i \in\{1, \ldots, n\}$, we have

$$
\left(F_{n} \circ g_{n} \circ F_{n}\right)\left(x_{1} \cdots x_{n}\right)=F_{n}\left(h_{1}\left(x_{1}\right) \cdots h_{n}\left(x_{n}\right)\right)=F_{n}\left(x_{1} \cdots x_{n}\right) .
$$

By Theorem 8, setting $H_{n}=g_{n} \circ F_{n}$ for every $n \geqslant 1$, there is a one-to-one function $f_{n}: \operatorname{ran}\left(H_{n}\right) \rightarrow Y$ such that $F_{n}=f_{n} \circ H_{n}$ and such that the map $H: X^{*} \rightarrow X^{*}$ obtained by setting $H_{0}(\varepsilon)=\varepsilon$ is associative and length-preserving. The conclusion follows from Example 3 .

(ii) $\Rightarrow$ (i) By Example 3 and Proposition 4 we obtain that $F$ is B-preassociative. Moreover, the kernel of $F$ is defined by the family $\left\{\operatorname{ker}\left(h_{i}\right): i \geqslant 1\right\}$ of equivalence relations on $X$. 


\section{ACKNOWLEDGMENTS}

This research is supported by the internal research project F1R-MTH-PUL-15MRO3 of the University of Luxembourg.

\section{REFERENCES}

[1] B. de Finetti. Sul concetto di media. Giornale dell' Instituto Italiano degli Attari 2(3):369-396, 1931.

[2] J. Fodor and M. Roubens. Fuzzy preference modelling and multicriteria decision support. Kluwer, Dordrecht, 1994.

[3] M. Grabisch, J.-L. Marichal, R. Mesiar, and E. Pap. Aggregation functions. Encyclopedia of Mathematics and its Applications, vol. 127. Cambridge University Press, Cambridge, 2009.

[4] A. N. Kolmogoroff. Sur la notion de la moyenne. (French). Atti Accad. Naz. Lincei, 12(6):388-391, 1930.

[5] E. Lehtonen, J.-L. Marichal, B. Teheux. Associative string functions. Asian-European Journal of Mathematics 7(4):1450059 (18 pages), 2014.

[6] J.-L. Marichal and B. Teheux. Associative and preassociative functions. Semigroup Forum 89(2):431-442, 2014. (Improved version available at arxiv.org/abs/1309.7303v3).

[7] J.-L. Marichal and B. Teheux. Preassociative aggregation functions. Fuzzy Sets and Systems 268:15-26, 2015.

[8] J.-L. Marichal and B. Teheux. Barycentrically associative and preassociative functions. Acta Mathematica Hungarica 145(2):468-488, 2015.

[9] M. Nagumo. Über eine Klasse der Mittelwerte. (German). Japanese Journ. of Math., 7:71-79, 1930.

[10] B. Schweizer and A. Sklar. Probabilistic metric spaces. North-Holland Series in Probability and Applied Mathematics. North-Holland Publishing Co., New York, 1983. (New edition in: Dover Publications, New York, 2005).

Mathematics Research Unit, FSTC, University OF LUXEMbourg, 6, RUE COUdENHOVE-KALERgi, L-1359 LUXEMBOURG, LUXEMBOURG

E-mail address: jean-luc.marichal [at] uni.lu

Mathematics Research Unit, FSTC, University of LuXembourg, 6, RUE COUdENHOVE-Kalergi, L-1359 LUXEMBOURG, LUXEMBOURG

E-mail address: bruno.teheux [at ] uni.lu 